\title{
Penile prosthesis implant with bi-triangular excision and graft for surgical therapy of Peyronie's disease: A case report
}

\author{
Alexandre de Freitas Miranda ${ }^{1}$, Bruno Lopes Cançado Machado ${ }^{2}$ \\ ${ }^{1}$ Reconstructive Urology and Andrology Section, Department of Urology, Ipanema Federal Hospital and Urogenital Research Unit, \\ State University of Rio de Janeiro, UERJ, Rio de Janeiro, Brazil; \\ ${ }^{2}$ Urological Private Practice, Rio de Janeiro, Brazil.
}

\begin{abstract}
Summary We present a case of a 65-year-old man, who presented with moderate erectile dysfunction and a dorsal penile deviation of $60^{\circ}$ caused by Peyronie's disease. The patient underwent bi-triangularshaped plaque excision, followed by grafting and implantation of inflatable penile prosthesis. Complete penile straightening, without mechanical or geometric abnormalities, was achieved using bi-triangular excision and grafting.

Postoperatively, the patient reported high satisfaction with the results and could perform sexual intercourse naturally. This novel technique corrects any degree of penile curvature, permits malleable and semi-rigid penile prosthesis implantation, avoids penile length loss, and eliminates additional incisions. To our knowledge, this case is the first in the literature in which the bi-triangular technique was successfully used for penile prosthesis implantation secondary to

Peyronie's disease. This new technique appears to be a good solution to correct penile curvature during penile prosthesis implantation for the treatment of Peyronie's disease associated with erectile dysfunction.
\end{abstract}

KEY WORDS: Peyronie's disease; Surgery; Graft; Penile prosthesis; Erectile dysfunction.

Submitted 30 June; Accepted 30 September

\section{INTRODUCTION}

Peyronie's disease (PD) is a connective tissue disorder characterized by tunica albuginea fibrosis that can generate penile deformity with curvature, narrowing, and shortening and, in $41 \%$ of cases, is associated with erectile dysfunction (ED). PD is often associated with psychological disturbance and has a male population prevalence of 3-9\%.

Penile prosthesis implantation (PPI) is a treatment for patients with PD and ED non-responsive to oral treatment. During the implantation, the surgeon may need to perform manual modeling, plaque incision if residual curvature after modeling is greater than $30^{\circ}$, and, if the defect created by the incision is greater than $2 \mathrm{~cm}$, placement of a graft. Segal et al. reported that $53.6 \%$ of patients underwent PPI and modeling required plaque incision with or without a graft (1). The graft insertion may represents a challenging procedure and in some cases require another incision to access distal defects. Herein we report the first case of PPI using the bi-triangular excision and graft (2).

\section{Case report}

Here we present a case of a 65-year-old man, without comorbidities, who presented with moderate ED and required a phosphodiesterase type 5 inhibitor to have sexual intercourse. He had a dorsal penile deviation of $60^{\circ}$ caused by PD. The penile plaque had been stable for 1 year. Artificial erection was induced with $0.9 \%$ of $\mathrm{NaCl}$ solution injected into the corpora cavernosa, punctured with a $21 G$ butterfly needle. The technique described by Austoni et al. was used to deglove the penis and isolate the neurovascular bundle (3). We marked two triangles on the patient's tunica albuginea. The bases of the triangles were equal to the disparity between the long and short sides of the curvature $(\mathrm{w})$, and the sum of the triangles' heights was equal to $3 / 4$ of the corpora cavernosa circumference (L) (Figure 1A). The apexes of the triangles were located at the middle of the curvature on the concave side (Figure 1A). After the excision of the triangles and rectification of the penis, the corpora cavernosa defect acquired a rectangular shape, with longitudinal and transversal lengths equal to $\mathrm{W}$ and $\mathrm{L}$, respectively (Figure 1B). The defect was closed by a bovine pericardium graft $4.5 \times 8.25 \mathrm{~cm}$ in size (Figure 1C). Finally the Buck's fascia was closed, and the penis re-gloved (Figure 1D). Another 2-cm longitudinal incision was made on each cavernous body to perform the PPI. The fluid reservoir was inserted into the retropubic space through an inguinal ring using the scrotal incision made to deglove the penis. The pump was placed on the subcutaneous space of the scrotum. A 14-Fr Blake drain was inserted into the scrotum for $24 \mathrm{~h}$.

The patient reassumed sexual intercourse 6 weeks after surgery. Complete penile straightening, without mechanical or geometric abnormalities, was achieved using bitriangular excision and grafting. Postoperatively, the patient reported being highly satisfied with the results and could perform sexual intercourse naturally.

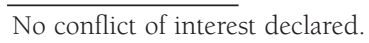


Figures 1a, b, $c$, $d$.

Surgical procedure.
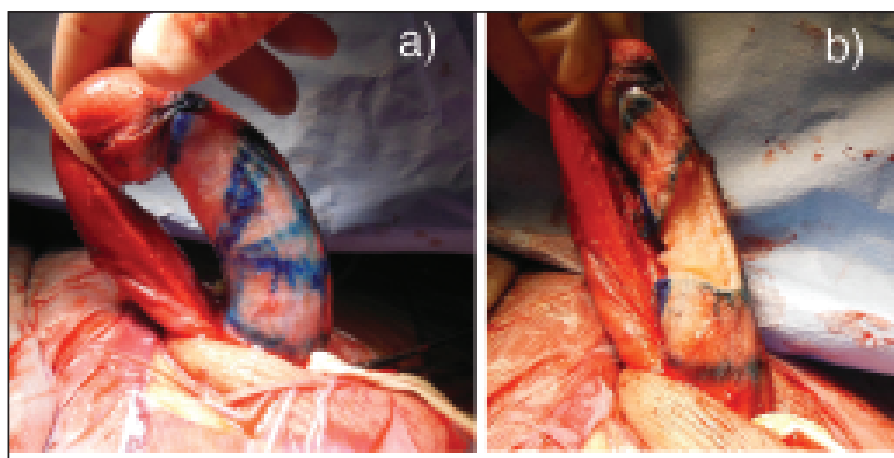

b)
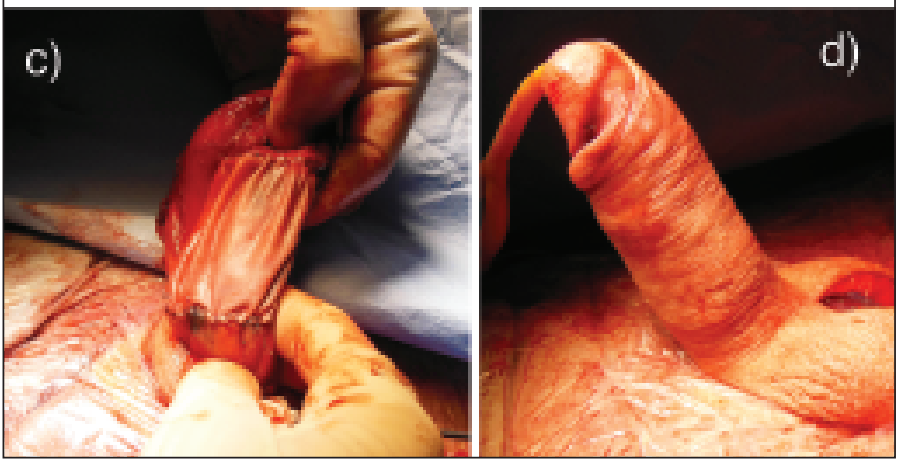

A) Penile degloving with the two triangles marked.

B) Defect resulting after bi-triangular excision.

C) Penile rectification and tunica albuginea defect closed by bovine pericardium graft.

D) Inflatable penile prosthesis implantation after re-gloving.

\section{Conclusions}

To our knowledge, this case is the first in the literature in which the previously reported bi-triangular technique was successfully applied for PPI secondary to PD, validating our previous measures and findings. This new technique appears to be a good solution to correct penile curvature during penile prosthesis implantation for the treatment of PD associated with ED.

Discussion and Supplementary References are posted as Supplementary Materials on www.aiua.it

\section{REFERENCES}

1. Segal RL, Cabrini MR, Bivalacqua TJ, Burnett AL. Penile straightening maneuvers employed during penile prosthesis surgery: technical options and outcomes. Int J Impot Res. 2014; 26:182-5.

2. Miranda AF, Sampaio FJ. A geometric model of plaque incision and graft for Peyronie's disease with geometric analyses of different techniques. J Sex Med. 2014; 11:1546-53.

3. Austoni E, Altieri VM, Tenaglia R. Trans-scrotal penile degloving, a new procedure for corporoplasties. Urologia. 2012; 79:200-10.

\section{Correspondence}

Alexandre de Freitas Miranda, MD (Corresponding Author) contato@alexandremiranda.com.br

Urogenital Research Unit, State University of Rio de Janeiro Av 28 de Setembro, 87, Fundos, Vila Isabel

Rio de Janeiro, RJ, Brazil, 20551-030

Bruno Lopes Cançado Machado, MD

blc1904@hotmail.com

Rua Visconde de Pirajá, 595, sala 1406, Ipanema

Rio de Janeiro, RJ, Brazil, 22410-002 